\title{
High-Performance Thin Film Composite Membranes for Energy Harvesting using Pressure Retarded Osmosis (PRO)
}

\author{
Dr. Mohtada Sadrzadeh \\ University of Alberta \\ Edmonton, AB Canada, T6G 1H9 \\ sadrzade@ualberta.ca
}

\begin{abstract}
The world is facing unprecedented challenges for water and energy supplies due to the rapid increase in consumption and depletion of these reserves and a reduction in the availability of low-cost fossil fuels. These concerns have motivated the development of technologies to reduce the use of fossil fuels and provide inexpensive, secure, and clean water and energy resources. Membrane technology has recently shown great potential for the sustainable production of both clean water and clean energy at the same time. Pressure retarded osmosis (PRO), known as "Blue Energy", is a membrane-based process that generates power from salinity gradients. PRO is part of the so-called osmotic power or salinity gradient energy sources, which is based on the release of free energy upon the mixing of two fluids with different salt concentrations, commonly freshwater and saltwater. The main component of the PRO technology is a semipermeable membrane that controls the overall power generation and profitability. The morphology and mechanical properties of the porous support layer was found to affect the performance of the PRO process significantly. The internal concentration polarization due to the accumulation of salt ions within the porous support increases the concentration of ions on the membrane surface and thus decreases the driving force significantly. This presentation outlines the strategies that can be used to fabricate more effective membrane substrates by optimizing their structural properties, such as thickness, porosity, tortuosity, and diffusivity of salt ions in the porous support, to mitigate concentration polarization in PRO membranes. These strategies include tuning the chemistry and pore structure of the support layer, incorporating novel nanomaterials and additives with high hydrophilic properties into the support layer, and developing stimuli-responsive antifouling support and the active layer. The objective was to achieve high-water permeability, low salt permeability, good mechanical strength, and optimum structural properties to generate a high power density during the PRO process.
\end{abstract}

